\title{
Use optimization of natural antioxidants in refined, bleached, and deodorized palm olein during repeated deep-fat frying using response surface methodology
}

\begin{abstract}
An optimization study on the use of oleoresin rosemary extract, sage extract, and citric acid added into refined, bleached, and deodorized (RBD) palm olein in deep-fat frying of potato chips was carried out using response surface methodology (RSM). Results showed that oleoresin rosemary extract was the most important factor affecting the sensory acceptability of potato chips. For taste and odor, its effects were highly significant $(\mathrm{P}<0.01)$, while for crispiness and overall acceptability, the effects were significant $(\mathrm{P}<0.05)$. As for sage extract, the level of this antioxidant had a highly significant $(\mathrm{P}<0.01)$ effect on appearance and taste and a significant effect $(\mathrm{P}<0.05)$ on odor and overall acceptability, but had no effect on crispiness. Although there was no significant synergistic correlation between citric acid and oleoresin rosemary extract or sage extract at the first order, its second order was significantly $(\mathrm{P}<0.05)$ related to taste, crispiness, and overall acceptability. An interaction between oleoresin rosemary and sage extracts also significantly $(\mathrm{P}<0.05)$ improved the score of overall acceptability of the potato chips. Contour maps of the sensory scores of potato chips indicated that the optimal points for appearance were achieved using $0.062 \%$ oleoresin rosemary extract, $0.066 \%$ sage extract, and $0.023 \%$ citric acid, while optimal task was achieved with $0.063 \%$ oleoresin rosemary extract, $0.075 \%$ sage extract, and $0.025 \%$ citric acid. With the same sequence of ingredients added into oil, the combinations required to achieve the optimal odor, crispiness, and overall acceptability scores were 0.058-0.046-0.026, 0.060-0.071-0.022, and 0.060-0.064-0.026\%, respectively.
\end{abstract}

Keyword: Citric acid; Deep-fat frying; Response surface methodology (RSM); Potato chips; RBD palm olein; Rosemary; Sage; Sensory evaluation 